\title{
Determinants of hospital costs in community-acquired pneumonia
}

\author{
S. Reyes**\#, R. Martinez*, J.M. Vallés`, E. Cases* and R. Menendez*,+
}

ABSTRACT: Community-acquired pneumonia (CAP) has a high incidence and involves an important consumption of healthcare resources. The present authors analysed the influence of comorbidity, initial severity and complications upon the direct costs associated with hospitalised CAP patients.

Direct hospitalisation costs (room cost, treatment, laboratory and diagnostic tests) were assessed in a prospective, observational study of 271 patients admitted to a hospital ward due to CAP.

The mean \pm SD patient age was $70 \pm 15$ yrs. The mortality rate was $11.1 \%$. Complications were found in $72.3 \%$ and comorbidities in $74.9 \%$. The median (interquartile range) total cost was $€ 1,683$ $(€ 1,291-2,471)$ and the component costs were: room cost $€ 1,286(€ 857-1,714)$; laboratory tests $€ 212$ (€171-272); treatment $€ 187$ (€114-304); and diagnostic procedures $€ 58$ (€29-122). Complications and higher Pneumonia Severity Index increased the costs, but age and comorbidity did not. A logistic regression analysis to predict high cost $(>€ 1,683)$ showed that infectious (odds ratio 6.8, 95\% confidence interval 1.3-36), digestive (5.9 (1.5-22.8)), pulmonary (2.6 (1.4-4.7)) and other complications (3.9 (1.8-8.4)) were independent risk factors, as were previous hospitalisation (2.3 (1.2-4.3)) and hypoalbuminaemia (2 (1.1-3.6)).

Complications, hypoalbuminaemia and previous hospitalisation were the main determinants of high direct costs of hospitalisation due to community-acquired pneumonia. Neither age nor comorbidities were independently associated with cost.

KEYWORDS: Community-acquired pneumonia, cost, length of stay, mortality, Pneumonia Severity Index, treatment

ommunity-acquired pneumonia (CAP) is a potentially serious disease with a high incidence and a large economic impact on both direct and indirect costs [1]. The main direct cost of the disease is due to hospitalisation of CAP patients, which can represent up to $90 \%$ of the global cost associated with CAP [2].

The global components of the direct costs of hospitalised CAP patients have been analysed recently [3,4]. The most important components are hospital stay and antibiotic treatment, both of which are influenced by initial disease severity and the clinical course followed by the patient during hospitalisation. However, in spite of the interest in determining the causes underlying the direct costs of hospitalised CAP patients and the adoption of measures designed to restrain these costs, to date few studies have conducted a detailed analysis of the impact of comorbidities and complications. Such information is important, since patients with comorbidities and/or complications are those most often hospitalised due to CAP. Furthermore, CAP is more frequent in elderly patients with comorbidities, and worsening of comorbidities is common. In turn, this prolongs the length of hospital stay (LOS) and increases the direct costs $[5,6]$. Conversely, an ageing population and increased survival among patients with chronic diseases lead to more complex CAP.

In recent years, a number of publications have analysed the economic aspects of CAP, aiming to evaluate costs in outpatients and in those admitted to the hospital or intensive care unit (ICU) [7, 8]. BAUER et al. [4] also found that direct costs correlated with initial severity as measured by the Pneumonia Severity Index (PSI) [9]. The present authors hypothesise that, in addition to these factors, the different components of direct costs are also influenced by comorbid conditions and complications, which would generate higher costs. Thus, identification of the determinants of high health costs could help define strategies for cost reduction or the more efficient use of existing resources [10-12].

The present study provides an analysis from the hospital perspective of the direct costs produced by the hospitalisation of CAP patients and
AFFILIATIONS

*Pneumology Service,

'Emergency Service, University

Hospital La Fe, Valencia, and

${ }^{\#}$ Dept of Medicine-Doctoral

Programme, Barcelona Autonomous University, Barcelona,

${ }^{+}$Centro de Investigación Biomédica en Red (CIBER), Ministry of Health, Spain.

CORRESPONDENCE

S. Reyes

Servicio de Neumología

Hospital Universitario La Fe

Avda. Campanar 21

46009 Valencia

Spain

Fax: 34961973089

E-mail: reyes@comv.es

Received:

August 092007

Accepted after revision:

December 112007

SUPPORT STATEMENT

This work received financial support from CIBER-CB06/06/0028.

STATEMENT OF INTEREST

None declared. 
evaluates the influence of advanced age, comorbidities, PSI and complications on the different cost components. In addition, the factors causing high costs of hospitalisation due to CAP are also analysed.

\section{PATIENTS AND METHODS}

\section{Study subjects}

A prospective observational study was carried out over 13 months in a public, tertiary care university hospital (University Hospital La Fe, Valencia, Spain).

The inclusion criteria were a clinical diagnosis compatible with CAP with two or more clinical symptoms and a new infiltrate on the chest radiograph. Patients were excluded if they: were aged <18 yrs; were immunodepressed (transplant patients, HIV-infected patients with a CD4+ count of $<100$ cells $\mu \mathrm{L}^{-1}$, patients receiving immunosuppressors and/or corticoids at doses of $>20 \mathrm{mg} \cdot$ day $^{-1}$ ); had lung abscesses; had been admitted to hospital in the previous 15 days; or had CAP requiring admission to the ICU.

The study was approved by the Local Ethics Committee (University Hospital La Fe). Informed consent was not necessary, since there were no interventions affecting either physicians' treatment decisions or the patients.

\section{Definitions}

Comorbidities were defined from previous studies $[9,13]$ as follows: cardiac (involving treatment for coronary artery disease, congestive heart failure or valvular heart disease); pulmonary (treatment for asthma, chronic obstructive pulmonary disease or interstitial lung disorders); renal (pre-existing kidney disease with documented abnormal serum creatinine levels outside the pneumonia episode); hepatic (pre-existing viral or toxic liver disease); central nervous system disorders (presence of symptomatic acute or chronic vascular or nonvascular encephalopathy, with or without dementia); diabetes mellitus (diagnosis of glucose intolerance and treatment with oral antidiabetic drugs or insulin); and neoplastic disease (any solid tumour active at the time of presentation or requiring antineoplastic treatment within the preceding year). Alcohol abuse was defined as the ingestion of an estimated amount of $>80 \mathrm{~g}$ alcohol $\cdot \mathrm{day}^{-1}$ for $\geqslant 1 \mathrm{yr}$ before presentation. Smokers were defined as current smokers of $>10$ cigarettes $\cdot$ day $^{-1}$ for at least the preceding year.

The classification of complications was also based on previous studies $[12,14]$ : pulmonary (respiratory failure, arterial oxygen tension $\left(\mathrm{Pa}, \mathrm{O}_{2}\right)<7.98 \mathrm{kPa}(<60 \mathrm{mmHg})$, pleural effusion, pulmonary embolism, pneumothorax); cardiovascular (congestive heart failure, cardiogenic shock, acute myocardial infarction, pericarditis); digestive (gastrointestinal bleeding, diarrhoea, altered liver function); renal (acute renal failure or exacerbation of chronic renal failure); infectious (empyema: evident pus in pleural space); nosocomial infection (endocarditis, arthritis, meningitis); and other complications (diabetic decompensation, leukopaenia, anaemia, water-electrolyte disorder, cerebrovascular stroke, etc.).

\section{Data collection}

The following data were collected: patient age and sex, smoking and alcohol habits, influenza vaccination, nursing home residency, prior hospitalisation, and comorbidities. The clinical symptoms recorded included cough, expectoration, pleuritic chest pain, dyspnoea, acute confusion, and signs such as temperature, rales, respiratory and cardiac frequencies, and systolic and diastolic blood pressure. Laboratory parameters were also recorded: leukocyte count, sodium, potassium, serum creatinine, plasma urea, glucose, albumin, aspartate aminotransferase/alanine aminotransferase, and arterial blood gases $\left(\mathrm{Pa}, \mathrm{O}_{2}\right.$, arterial carbon dioxide tension and $\left.\mathrm{pH}\right)$. Initial severity was calculated with the PSI [9]. The following evolutive parameters were recorded: complications, LOS, and mortality after 30 days.

\section{Cost calculations}

The cost study was carried out from the hospital perspective. All the direct costs during hospitalisation were analysed, i.e. those related to the consumption of healthcare resources, such as diagnostic and laboratory tests, treatment and LOS. The indirect costs associated with work days missed or transport to hospital were not considered.

The tests performed during hospitalisation were quantified and classified into five groups as follows. 1) Blood tests: complete blood counts, biochemistry, haemostasis, blood gas tests. 2) Microbiological studies: blood and sputum cultures, viral serology and atypical bacteria, urinary antigens of Legionella pneumophila and Streptococcus pneumoniae. 3) Radiological studies: plain radiographs, computed tomography. 4) Endoscopic and invasive techniques: bronchoscopy, thoracocentesis, chest drainage. 5) ECGs.

The treatment provided was classified as either antibiotic treatment or other treatment. Other treatments included the treatment of comorbidities, concomitant treatment and treatment of complications. In relation to each drug used, the number of doses administered and the route of administration (oral, intravenous, intramuscular, subcutaneous or inhaled) were documented.

The LOS in hospital was calculated as the number of days of admission, from arrival at the hospital until the day of discharge.

The calculated costs were grouped as follows: 1) cost of medication, subclassified as cost of antibiotic treatment and cost of other treatments; 2) room cost; 3) cost of laboratory tests (blood tests and microbiological studies); and 4) cost of diagnostic tests (radiological studies, endoscopic and invasive techniques, and ECGs). The total hospital cost was calculated as the sum of the categories above. All costs were calculated in Euros $(€)$ for the year 2002. The medication costs were calculated as the median cost of the drugs for the hospital during 2002 (data provided by the Pharmacy Service of the University Hospital $\mathrm{La} \mathrm{Fe}$ ). The costs of the radiological, microbiological and other relevant tests were obtained from the official fees of the Servicio Valenciano de Salud [15] (table 1). The cost per day of hospital stay was provided by the Servicio Valenciano de Salud for the study hospital [15].

\section{Statistical analysis}

The cost results are expressed as median (interquartile range). Qualitative variables were compared using the Chi-squared test, and quantitative variables were compared using an unpaired t-test or the Mann-Whitney U-test, where 


\section{TABLE 1 Cost of diagnostic tests}

\begin{tabular}{lr} 
& Cost (€) \\
\hline Blood tests & \\
Complete blood count & 6.74 \\
Biochemistry & 21.40 \\
Haemostasis & 15.00 \\
Blood gases & 30.00 \\
Microbiological tests & \\
Blood culture & 15.52 \\
Sputum culture, bronchial aspirate & 13.39 \\
Serology, urine antigens & 5.85 \\
Imaging techniques & \\
Chest or abdominal radiographs & 9.23 \\
CT & 71.10 \\
ECG & 3.93 \\
Invasive techniques & \\
Bronchoscopy & 82.94 \\
Thoracocentesis & 54.00 \\
Chest drainage & 95.60 \\
\hline
\end{tabular}

appropriate. The correlation between variables was analysed using Spearman correlation analysis. Differences in quantitative variables were assessed by ANOVA or with a KruskalWallis test, as appropriate. A p-value of $<0.05$ was considered significant.

Uni- and multivariate statistical analyses were carried out for the clinical variables, comorbidities and complications registered in the protocol, and for high cost of CAP. High cost was defined as cost in excess of the median of the cohort $(>€ 1,683)$ and was the dependent variable in a stepwise logistic regression analysis. Independent variables were those found to be significant in the univariate analysis and others considered clinically relevant, such as comorbid conditions and advanced age. Independent variables were introduced in the model and dichotomised as follows: advanced age ( $\geqslant 70$ yrs: yes/no); respiratory failure $\left(\mathrm{Pa}_{1} \mathrm{O}_{2}<7.98 \mathrm{kPa}(<60 \mathrm{mmHg})\right.$ : yes $\left./ \mathrm{no}\right)$; hypoalbuminaemia $\left(\leqslant 3 \mathrm{mg} \cdot \mathrm{dL}^{-1}\right.$ : yes $/$ no); raised urea $(\leqslant 42 \mathrm{mg}$. $\mathrm{dL}^{-1}$ : yes $\left./ \mathrm{no}\right)$, and hyperglycaemia $\left(\leqslant 129 \mathrm{mg} \cdot \mathrm{dL}^{-1}\right.$ : yes $\left./ \mathrm{no}\right)$. PSI was categorised into high-risk classes (IV, V) or low-risk classes (I-III). The Hosmer and Lemeshow goodness-of-fit test was performed for each model [16].

\section{RESULTS}

\section{Study population}

The study included 271 patients hospitalised due to CAP, whose demographic characteristics, comorbidity, PSI [9], complications and mortality are reported in table 2 . An aetiological diagnosis was reached in $35(12.9 \%)$ patients; the most frequently found microorganisms were: S. pneumoniae $(\mathrm{n}=22,8.1 \%)$, Enterococcus faecalis $(\mathrm{n}=3,1.1 \%)$, Staphylococcus aureus $(\mathrm{n}=2,0.7 \%)$, Pseudomonas aeruginosa $(\mathrm{n}=2,0.7 \%)$, Haemophilus parainfluenzae $(\mathrm{n}=2,0.7 \%)$, L. pneumophila $(\mathrm{n}=1,0.4 \%)$, and other microorganisms $(n=4,1.5 \%)$. Bacteraemia was detected in $16(5.9 \%)$ patients. Comorbidity was found in $203(74.9 \%)$ patients, a single comorbid condition existed in $122(45 \%)$ patients, and two or more existed in $81(29.9 \%)$ patients. Complications, shown in table 2, appeared in 196 (72.3\%) patients. Among the pulmonary complications, $100(36.9 \%)$ patients presented with respiratory failure, and $42(17 \%)$ presented with pleural effusion. Cardiovascular complications were congestive heart failure $(n=41,15.1 \%)$ and cardiogenic shock $(n=10,3.7 \%)$; digestive complications were liver disorders $(n=7,2.6 \%)$, bleeding $(n=6$, $2.2 \%)$ and diarrhoea $(n=9,3.3 \%)$; infectious complications comprised nosocomial infection $(n=6,2.2 \%)$, empyema $(n=5$, $1.8 \%)$, meningitis $(n=1,0.4 \%)$ and endocarditis $(n=1,0.4 \%)$. In total, $30(11.1 \%)$ patients died.

\section{Cost results}

The median (interquartile range; IQR) total cost of the cohort was $€ 1,683(€ 1,291-2,471)$. The distribution of each component was as follows: room cost $€ 1,286$ (€857-1,714); laboratory tests $€ 212(€ 171-272)$; treatment $€ 187(€ 114-304$; antibiotic treatment $€ 138(€ 81-229)$ and other treatments $€ 38(€ 17-68))$; and diagnostic procedures $€ 58(€ 29-122)$. The percentage of the

TABLE $2 \begin{aligned} & \text { Demographic characteristics, comorbidity, } \\ & \text { Pneumonia Severity Index (PSI), length of stay } \\ & \text { (LOS), complications and mortality during } \\ & \text { hospital admission }\end{aligned}$
Characteristics

\begin{tabular}{|c|c|}
\hline Subjects n & 271 \\
\hline Age yrs & $70 \pm 15$ \\
\hline $\operatorname{Sex} M / F$ & $161(59.4) / 110(40.6)$ \\
\hline Smoker & $41(15.1)$ \\
\hline Alcohol abuse & $41(15.1)$ \\
\hline Nursing home & $8(3.0)$ \\
\hline \multicolumn{2}{|l|}{ Comorbidity } \\
\hline Neoplasm & $13(4.8)$ \\
\hline Liver disease & $9(3.3)$ \\
\hline Cardiovascular disease & 89 (32.8) \\
\hline Cerebrovascular disease & $31(11.4)$ \\
\hline Kidney disease & $16(5.9)$ \\
\hline Diabetes & $57(21.0)$ \\
\hline COPD & 69 (25.5) \\
\hline \multicolumn{2}{|l|}{ PSI } \\
\hline 1 & $22(8.1)$ \\
\hline$\|$ & $36(13.3)$ \\
\hline III & $53(19.6)$ \\
\hline IV & $113(41.7)$ \\
\hline V & 47 (17.3) \\
\hline LOS median days & 9 \\
\hline \multicolumn{2}{|l|}{ Hospital complications } \\
\hline Pulmonary & $142(52.4)$ \\
\hline Renal & $21(7.7)$ \\
\hline Cardiovascular & $51(18.8)$ \\
\hline Digestive & $22(8.1)$ \\
\hline Infectious & $13(4.8)$ \\
\hline Others & 55 (20.3) \\
\hline Mortality & $30(11.1)$ \\
\hline
\end{tabular}

Data are presented as mean \pm SD or $n(\%)$, unless otherwise stated. M: male F: female; COPD: chronic obstructive pulmonary disease. 
total cost corresponding to each component was as follows: room cost $69.3 \%$, laboratory tests $11.9 \%$, treatment $13.1 \%$, and diagnostic procedures $5.7 \%$.

The median (IQR) total cost in patients with an aetiological diagnosis was significantly greater than that of patients with an unknown diagnosis $(€ 2,102(€ 1,485-3,485)$ versus $€ 1,645$ $(€ 1,261-2,286) ; p=0.01)$. Of these, the components with significantly greater costs were: laboratory tests $(€ 265$ (€190-317) versus $€ 205$ (€168-262); $\mathrm{p}=0.001)$; diagnostic procedures (€107 (€42234) versus $€ 53(€ 29-116) ; \mathrm{p}=0.009)$; and room cost $(€ 1,714$ $(€ 1,000-2,000)$ versus $€ 1,143(€ 857-1,714) ; \mathrm{p}=0.01)$. No significant differences were found in the costs according to the causal microorganism. However, the median (IQR) total cost of bacteraemia patients was significantly higher compared with nonbacteraemia patients $(€ 2,083(€ 1,507-3,775)$ versus $€ 1,628$ $(€ 1,231-2,402) ; p=0.04)$. When these were broken down into components, the costs were greater in laboratory tests $(€ 256$ $(€ 196-384)$ versus $€ 213$ (€175-271); $\mathrm{p}=0.049)$ and room cost $(€ 1,714(€ 1,143-2,429)$ versus $€ 1,143(€ 857-1,714) ; \mathrm{p}=0.03)$.

The costs according to patient age, sex, influenza vaccination, comorbidity and complications are described and compared in table 3. A tendency towards increased cost was recorded among patients $>70$ yrs of age, although statistical significance was not reached. The influenza vaccination was not significantly related to initial severity, LOS or costs. The median (IQR) total cost of patients without comorbidity, with a single comorbidity and with two or more comorbidities were $€ 1,631$ $(€ 1,304-2,218), € 1,637(€ 1,180-2,320)$ and $€ 1,771(€ 1,386-2,695)$, respectively $(\mathrm{p}=0.03)$.
The direct health costs according to the PSI are reported in table 4. A positive correlation was found between the total cost (rho $=0.15, \mathrm{p}=0.009)$ as well as the cost of each component, except diagnostic studies, and the PSI, i.e. the higher the risk class, the greater the cost.

The median (IQR) total cost of uncomplicated pneumonias was $€ 1,295(€ 1,055-1,637)$ versus $€ 1,692(€ 1,392-2,083)$ in patients with a single complication, and $€ 2,111(€ 1,485-3,007)$ in patients with two or more complications $(p=0.0001$; table 5$)$. The differences were significant for each of the cost components. The total costs according to the type of complication are depicted in table 3; infectious, renal and cardiovascular complications had the highest costs.

The median total cost in survivors was $€ 1,690$ (€1,294-2,313), and in nonsurvivors $€ 1,574$ ( $€ 978-3,476 ; \mathrm{p}=0.9)$. There were significant differences in the following components of the cost between survivors and nonsurvivors: treatment $(€ 177(€ 113-281)$ versus $€ 353$ (€123-584); $\mathrm{p}=0.004)$; nonantibiotic treatment $(€ 33$ (€15-66) versus $€ 63$ (€23-271); $\mathrm{p}=0.001)$; and laboratory costs $(€ 205(€ 168-265)$ versus $€ 260(€ 204-298) ; \mathrm{p}=0.003)$. However, no difference in the cost of hospital stay was found (€1,286 (€857$1,714)$ versus $€ 1,000(€ 429-2,286) ; \mathrm{p}=0.1)$, although the LOS was greater for the survivors (median 9 days) than for the nonsurvivors (median 7 days).

\section{Factors related to high cost}

Univariate analysis

Advanced patient age, sex, toxic habits, nursing home residency and comorbidities were not significantly associated

TABLE 3 Hospital costs according to patient age and sex, and presence of comorbidities and complications

\begin{tabular}{|c|c|c|c|}
\hline Presence of comorbidities/complications & Yes & No & p-value ${ }^{\#}$ \\
\hline Males & $1636(1177-2392)$ & $1812(1370-2533)$ & 0.2 \\
\hline Influenza vaccination & $1796(1371-2483)$ & $1547(1333-2786)$ & 0.6 \\
\hline \multicolumn{4}{|l|}{ Comorbidities } \\
\hline Neoplasm & $1291(1030-2059)$ & $1691(1328-2513)$ & 0.1 \\
\hline Central nervous system & $1833(1291-2730)$ & $1655(1278-2354)$ & 0.2 \\
\hline Renal disease & $1696(1337-2433)$ & $1674(1273-2471)$ & 0.7 \\
\hline Diabetes & $1700(1378-2715)$ & $1665(1271-2367)$ & 0.4 \\
\hline COPD & $1771(1320-2674)$ & $1658(1271-2274)$ & 0.2 \\
\hline \multicolumn{4}{|l|}{ Complications } \\
\hline Pulmonary & $1996(1394-2843)$ & $1485(1122-1979)$ & 0.001 \\
\hline Empyema & $2075(1531-2892)$ & $1666(1287-2427)$ & 0.3 \\
\hline Nosocomial infection & $3405(1728-5716)$ & $1659(1282-2392)$ & 0.06 \\
\hline Cardiovascular & $2691(1375-3485)$ & $1634(1261-2154)$ & 0.002 \\
\hline Digestive & $2591(1755-3807)$ & $1634(1263-2275)$ & 0.002 \\
\hline Renal & $2744(1414-3616)$ & 1655 (1271-2292) & 0.04 \\
\hline Others & 2068 (1516-3607) & $1620(1182-2223)$ & 0.0001 \\
\hline
\end{tabular}

Data are presented as median (interquartile range) cost in Euros, unless otherwise stated. COPD: chronic obstructive pulmonary disease. ${ }^{*}$ : Mann-Whitney U-test. 
TABLE 4 Direct costs and Pneumonia Severity Index (PSI)

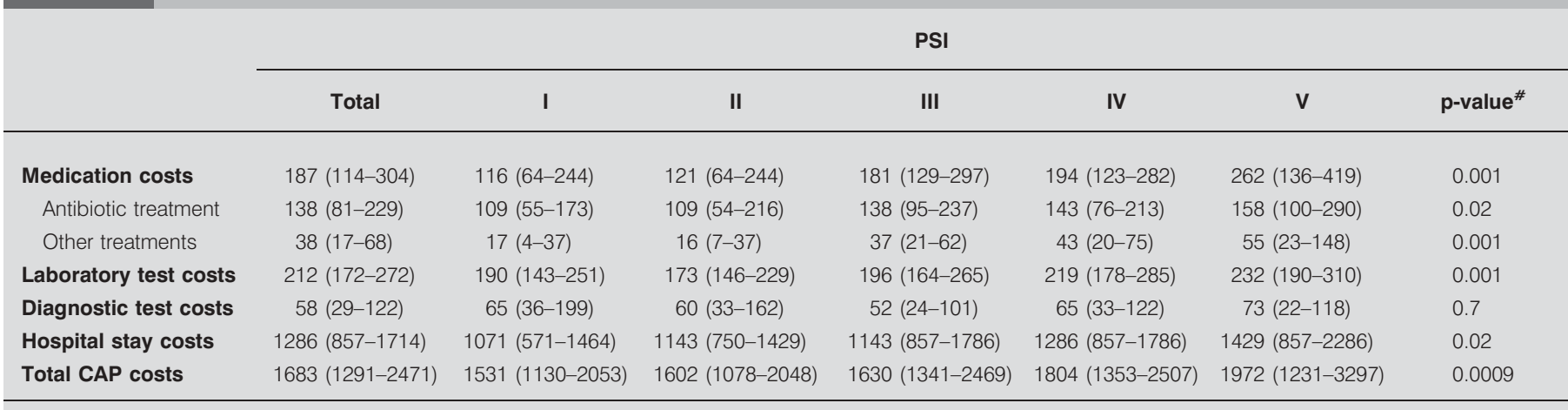

Data are presented as median (interquartile range) cost in Euros, unless otherwise stated. CAP: community-acquired pneumonia. ${ }^{\#}$ : Spearman correlation

with high cost in CAP. In contrast, prior hospitalisation was associated with higher cost (median $€ 1,624$ versus $€ 1,888$; $\mathrm{p}=0.01$ ). A higher PSI was also associated with high cost $(p=0.01 ;$ table 4$)$. The laboratory test disorders found in patients with high costs were: raised urea $(\mathrm{p}=0.01)$, hyperglycaemia $(p=0.007)$, respiratory failure $(p=0.002)$, and hypoalbuminaemia $(p=0.0001)$. The median cost of all complications (pulmonary, infectious, cardiovascular, digestive, renal and others) was significantly associated with high cost $(p=0.0001)$.

\section{Multivariate analysis}

The following independent variables were included in the model because they proved significant in the univariate analysis: prior hospitalisation, respiratory failure, hypoalbuminaemia, raised urea, hyperglycaemia, PSI and complications. Advanced age and comorbidities (found to be nonsignificant) were also included because they were study objectives. The mathematical model selected the following variables as being associated with high cost (table 6): infectious, digestive, pulmonary and other complications, hypoalbuminaemia and prior hospital admission. The Chi-squared goodness-of-fit analysis demonstrated the adequacy of the model $(\mathrm{p}>0.05)$.

\section{DISCUSSION}

The most relevant findings of the present study can be summarised as follows. 1) The median (IQR) total cost of hospitalised CAP patients was $€ 1,683(€ 1,291-2,471)$, and LOS generated the highest direct cost $(69.3 \%)$. 2) Complications, particularly of an infectious nature, significantly and independently increased the direct costs of hospitalised CAP patients. 3) Neither patient age nor comorbidities was independently associated with high cost. 4) Hospital admission in the preceding year and hypoalbuminaemia also raised costs.

The annual financial burden of pneumonia was estimated by the European Respiratory Society to be $€ 10.1$ billion in Europe; in-patient care costs accounted for $€ 5.7$ billion of this amount [1]. In Spain, MONGE and co-workers [17, 18] recorded 53,000 annual hospital admissions due to CAP, with an annual healthcare cost of $\sim € 115$ million. In the present study, the documented costs were slightly higher than those reported in the national and European literature. In other studies carried out in Spain, the direct costs of in-patient care were $€ 1,210$ [8] and $€ 1,553$ [7]. In Europe, the direct costs ranged from $€ 1,333$ in Germany [4] to $€ 2,550-7,650$ in the UK [19]. Nevertheless, the European costs were significantly lower than those reported for hospitals in the USA, despite longer average stays $[3,20,21]$. In a prospective study of four hospitals in the USA, FINE et al. [3] recorded a median total cost of $€ 4,468$ for hospitalised CAP patients.

Hospital admission due to CAP is dependent upon a number of factors, such as PSI, comorbidities and complications. In elderly subjects, admission is decided based on the associated comorbidities and complications, both of which are more frequent in this population group [21-23]. However, in young adults, an increased probability of admission is determined by the presence of serious complications and, to a lesser extent, by comorbidities $[22,24]$.

The contribution of each of these factors, comorbidities, PSI and complications, to the total cost of hospitalised CAP patients has not been extensively investigated, although previous studies have analysed the effect of patient age. BARTOLOMÉ et al. [7] reported significantly higher costs in patients $>65$ yrs of age, although no details were provided on the impact of severity or comorbidities. Likewise, NIEDERMAN et al. [23] also found in-patient cost in patients aged $>65 \mathrm{yrs}$ to reach US\$305 million versus US\$192 million in those $<65$ yrs of age. This difference in cost was fundamentally attributable to the higher percentage of admissions and the longer duration of stay for more elderly subjects. However, the impact of comorbidity on cost was only confirmed in those patients requiring respiratory services, possibly in relation to the longer LOS. While the results of the present study show a tendency towards increased cost among patients aged $>70 \mathrm{yrs}$, the difference did not reach statistical significance, and age was not selected as an independent variable by the multivariate analysis. Moreover, the presence of two or more comorbidities did not cause a significant increase in hospital costs.

In the present study, an increase in total cost was shown for patients with increased severity of CAP, and for patients with more complications. BAUER et al. [4] reported greater costs for patients with severe pneumonia requiring admission to ICU, compared with patients with less severe pneumonia, mainly due to longer LOS in hospital (US\$2,300 versus US $\$ 1,242 ; \mathrm{p}<0.001$ ). KAPLAN et al. [22] also found patients with complicated CAP 
TABLE 5 Direct costs of community-acquired pneumonia (CAP) according to the presence of complications

\begin{tabular}{cccc} 
& Complications & & p-value \\
\hline 0 & 1 & $\geqslant 2$
\end{tabular}

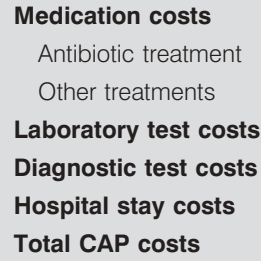

Medication costs

Antibiotic treatment

Laboratory test costs

Diagnostic test costs

Total CAP costs

$\begin{aligned} 115 & (64-155) \\ 20 & (8-39) \\ 174 & (153-212) \\ 42 & (22-69) \\ 1000 & (714-1286) \\ 1295 & (1055-1637)\end{aligned}$

$139(90-222)$
$36(18-56)$
$219(178-277)$
$53(19-84)$
$1286(1000-1571)$
$1692(1392-2083)$

$155(87-290)$
$57(23-130)$
$239(189-310)$
$97(33-218)$
$1429(1000-2286)$
$2111(1485-3007)$

0.0001

0.0001

0.0001

0.0001

0.0001

0.0001

Data are presented as median (interquartile range) cost in Euros, unless otherwise stated. ${ }^{*}$ : Kruskal-Wallis test.

(admission to ICU or the need for mechanical ventilation) to require longer hospital stays, resulting in increased costs compared with patients with uncomplicated CAP (US\$8,725 versus US $\$ 3,754)$. Although the present study did not include patients admitted to the ICU, an increased cost in patients with a high PSI, due to an increased number of tests and a longer LOS, was confirmed. KAPLAN et al. [22] also found that patients $>65 \mathrm{yrs}$ of age with comorbidity presented more complications. However, this finding is also explained by the fact that the PSI was higher in this group.

The percentage of complicated pneumonias in the present cohort of patients was high $(72.3 \%)$, similar to the figures reported in the literature [14]. Infectious complications caused the highest direct total costs, particularly nosocomial infection $(€ 3,405)$. It is well known that, while the incidence of nosocomial infection is low in CAP, it is a cause of treatment failure and seriously worsens the prognosis [13]. The present authors found this complication to increase the cost up to sixfold, due to the need for more tests and treatments, and, especially, due to a prolongation of LOS [25]. However, the absolute impact of pulmonary, cardiac and renal complications on the global cost of CAP was higher, since they produced significant cost increases, and mainly because their incidence was much higher. Furthermore, it was not uncommon to find more than one complication, a condition which in turn generated a greater cost increase.

TABLE 6 Independent factors associated with high cost ${ }^{\#}$ OR $(95 \% \mathrm{Cl})$

Previous hospital admission
Hypoalbuminaemia
Infectious complications
Digestive complications
Pulmonary complications
Other complications

$2.3(1.2-4.3)$
$2.0(1.1-3.6)$
$6.8(1.3-36)$
$5.9(1.5-22.8)$
$2.6(1.4-4.7)$
$3.9(1.8-8.4)$

OR: odds ratio; Cl: confidence interval. ${ }^{\#}$ : more than median total cost $(€ 1,683)$ of community-acquired pneumonia.
The multivariate analysis confirmed that infectious (odds ratio (OR) 6.8), digestive (OR 5.9), pulmonary (OR 2.6) and other complications (OR 3.9) are the strongest independent risk factors for high cost. In addition, two other variables with independent effects were identified in the mathematical model: hypoalbuminaemia (OR 2.0) and previous hospital admission (OR 2.3). Low plasma albumin is associated with more severe CAP, and is common in the elderly. It contributes to a slower clinical response to treatment and a poorer prognosis [6, 26]. CABRÉ et al. [27] found prior hospitalisation to be a predictor of poor patient course and increased mortality after 30 days. One of the possible explanations for this is that prior hospitalisation is more frequent in older patients [28]. The advanced age predisposes them to more serious conditions, the appearance of more complications and greater mortality $[27,29,30]$. However, the existing literature does not show a direct relationship between prior hospitalisation and hospital costs, although all of these factors produce an increase in the total cost of the CAP. Curiously, neither age nor comorbidity was independently associated with increased inpatient cost. Similar observations apply to PSI, the effect of which also disappeared in the multivariate analysis. Thus, the hospital cost of CAP was not significantly increased in patients with comorbidities, or even with a high PSI, if they had no associated complications.

In conclusion, complications, hypoalbuminaemia and previous hospital admission were the most important determinants of high cost associated with hospitalised community-acquired pneumonia patients. However, neither patient age nor comorbidities contributed to raising the direct costs. The Pneumonia Severity Index alone was not an independent risk factor for high cost either. Strategies designed to prevent complications during hospital stay, and early management of the complications that appear, may contribute to reducing in-patient costs in hospitalised cases of community-acquired pneumonia.

\section{REFERENCES}

1 Ambrosino N, Armaganidis A, Beurskens H. Pneumonia. In: European Respiratory/European Lung Foundation. European Lung White Book. The First Comprehensive Survey on Respiratory Health in Europe. Loddenkemper R, 
Gibson GJ, Sibille Y, eds. Sheffield, UK, ERSJ, 2003; pp. 55-65.

2 Niederman MS, Mandell LA, Anzueto A, et al. Guidelines for the management of adults with community-acquired pneumonia. Diagnosis, assessment of severity, antimicrobial therapy, and prevention. Am J Respir Crit Care Med 2001; 163: 1730-1754.

3 Fine MJ, Pratt HM, Obrosky DS, et al. Relation between length of hospital stay and costs of care for patients with communityacquired pneumonia. Am J Med 2000; 109: 378-385.

4 Bauer TT, Welte T, Ernen C, et al. Cost analyses of communityacquired pneumonia from the hospital perspective. Chest 2005; 128: 2238-2246.

5 Masotti L, Ceccarelli E, Cappelli R, Barabesi L, Forconi S. Community-acquired pneumonia in elderly patients and length of hospitalization. Arch Intern Med 2000; 160: 2678-2679.

6 Menéndez R, Cremades MJ, Martinez-Moragón E, Soler JJ, Reyes S, Perpiñá M. Duration of length of stay in pneumonia: influence of clinical factors and hospital type. Eur Respir J 2003; 22: 643-648.

7 Bartolomé M, Almirall J, Morera J, et al. A populationbased study of the costs of care for community-acquired pneumonia. Eur Respir J 2004; 23: 610-616.

8 González-Moraleja J, Sesma P, González C, López ME, Garcia JF, Alvarez-Sala JL. [What is the cost of inappropriate admission of pneumonia patients?]. Arch Bronconeumol 1999; 35: 312-316.

9 Fine MJ, Auble TE, Yealy DM, et al. A prediction rule to identify low-risk patients with community-acquired pneumonia. N Engl J Med 1997; 336: 243-250.

10 Torres A, Menéndez R. Mortality in COPD patients with community-acquired pneumonia: who is the third partner? Eur Respir J 2006; 28: 262-263.

11 Rhew DC, Tu GS, Ofman J, Henning JM, Richards MS, Weingarten SR. Early switch and early discharge strategies in patients with community-acquired pneumonia: a metaanalysis. Arch Intern Med 2001; 161: 722-727.

12 Weingarten SR, Riedinger MS, Hobson P, et al. Evaluation of a pneumonia practice guideline in an interventional trial. Am J Respir Crit Care Med 1996; 153: 1110-1115.

13 Arancibia F, Ewig S, Martinez JA, et al. Antimicrobial treatment failures in patients with community-acquired pneumonia: causes and prognostic implications. Am J Respir Crit Care Med 2000; 162: 154-160.

14 Fine MJ, Stone RA, Singer DE, et al. Processes and outcomes of care for patients with community-acquired pneumonia: results from the Pneumonia Patient Outcomes Research Team (PORT) cohort study. Arch Intern Med 1999; 159: 970-980.

15 Diari Oficial de la Generalitat Valenciana. www.docv. gva.es/portal/portal/2002/12/31/pdf/doc/2002_14538.pdf Date last accessed: May 31, 2003. Date last updated: December 31, 2002.
16 Hosmer D, Lemeshow S, eds. Applied Logistic Regression. New York, John Wiley \& Sons, 1989.

17 Monge V, San-Martin VM, González A. The burden of community-acquired pneumonia in Spain. Eur J Public Health 2001; 11: 362-364.

18 Monge V, González A. Hospital admissions for pneumonia in Spain. Infection 2001; 29: 3-6.

19 Guest JF, Morris A. Community-acquired pneumonia: the annual cost to the National Health Service in the UK. Eur Respir J 1997; 10: 1530-1534.

20 Lave JR, Fine MJ, Sankey SS, Hanusa BH, Weissfeld LA, Kapoor WN. Hospitalized pneumonia. Outcomes, treatment patterns, and costs in urban and rural areas. J Gen Intern Med 1996; 11: 415-421.

21 Colice GL, Morley MA, Asche C, Birnbaum HG. Treatment costs of community-acquired pneumonia in an employed population. Chest 2004; 125: 2140-2145.

22 Kaplan V, Angus DC, Griffin MF, Clermont G, Scott Watson R, Linde-Zwirble WT. Hospitalized communityacquired pneumonia in the elderly: age- and sex-related patterns of care and outcome in the United States. Am J Respir Crit Care Med 2002; 165: 766-772.

23 Niederman MS, McCombs JS, Unger AN, Kumar A, Popovian R. The cost of treating community-acquired pneumonia. Clin Ther 1998; 20: 820-837.

24 Rosón B, Carratalà J, Dorca J, Casanova A, Manresa F, Gudiol F. Etiology, reasons for hospitalization, risk classes, and outcomes of community-acquired pneumonia in patients hospitalized on the basis of conventional admission criteria. Clin Infect Dis 2001; 33: 158-165.

25 Menéndez R, Torres A, Rodriguez de Castro F, et al. Reaching stability in community-acquired pneumonia: the effects of the severity of disease, treatment, and the characteristics of patients. Clin Infect Dis 2004; 39: 1783-1790.

26 Riquelme R, Torres A, el-Ebiary M, et al. Communityacquired pneumonia in the elderly. Clinical and nutritional aspects. Am J Respir Crit Care Med 1997; 156: 1908-1914.

27 Cabré M, Serra-Prat M, Bolíbar I, Pallarés R. [Prognostic factors of community acquired pneumonia in very old patients.]. Med Clin (Barc) 2006; 127: 201-205.

28 Saldías Peñafiel F, O’Brien Solar A, Gederlini Gollerino A, Farías Gontupil G, Díaz Fuenzalida A. [Communityacquired pneumonia requiring hospitalization in immunocompetent elderly patients: clinical features, prognostic factors and treatment.]. Arch Bronconeumol 2003; 39: 333-340.

29 Mandell LA, Wunderink RG, Anzueto A, et al. Infectious Diseases Society of America/American Thoracic Society consensus guidelines on the management of communityacquired pneumonia in adults. Clin Infect Dis 2007; 44: Suppl. 2, S27-S72.

30 Bont J, Hak E, Hoes AW, Schipper M, Schellevis FG, Verheij TJ. A prediction rule for elderly primary-care patients with lower respiratory tract infections. Eur Respir $J$ 2007; 29: 969-975. 